\title{
Combined Mutation of the GATA2 Gene and STAT5B Gene in a Patient with Hypogammaglobulinemia and Autoimmunity
}

\author{
Zhihui Liu, ${ }^{1}$ Pingying Qing, ${ }^{1}$ Yi Zhao, ${ }^{1}$ Yi Liu ${ }^{1}$ and Tony N. Marion ${ }^{1,2}$ \\ ${ }^{1}$ Department of Rheumatology and Immunology, West China Hospital, Sichuan University, Chengdu, Sichuan, \\ China \\ ${ }^{2}$ Department of Microbiology, Immunology, and Biochemistry, University of Tennessee Health Science Center, \\ Memphis, TN, USA
}

\begin{abstract}
Antibody deficiency is a type of primary immunodeficiency that often manifests as primary hypogammaglobulinemia, with or without repeated infections. Although primary immunodeficiency appears to be contrary to autoimmunity, they usually occur simultaneously, and the specific pathogenesis remains unknown. We herein describe an adult patient with autoimmune manifestations and recurrent infections. The case was characterized by a sustained decrease in serum immunoglobulin A, accompanied by decreased $T$ lymphocytes, B lymphocytes, monocytes, and platelets in the peripheral blood and the presence of antinuclear and anti-SSA antibodies. Whole-exome sequencing for the patient revealed two spontaneous mutations in GATA2 (c.1084C>T) and STAT5B (c.1924A>C). This case report provides evidence that mutations in the GATA2 and STAT5B genes may be pathogenic in primary immunodeficiency and provides genetic evidence for the possible pathogenesis of primary immunodeficiency with autoimmune symptoms. However, further studies are needed to confirm the causal relationship.
\end{abstract}

Keywords: antibody deficiency; autoimmunity; gene mutations; primary immunodeficiency; whole-exome sequencing

Tohoku J. Exp. Med., 2021 October, 255 (2), 143-146.

\section{Introduction}

Antibody deficiency comprises a group of primary immunodeficiency diseases in which the production of antibodies is impaired due to inherent molecular defects of $\mathrm{B}$ lymphocytes or a lack of interaction between B lymphocytes and T lymphocytes (Notarangelo et al. 2004). Clinical features include repeated infections of the upper and lower respiratory and gastrointestinal tracts, accompanied by a decrease in the level of one or more serum immunoglobulins, as represented by $\operatorname{IgG}, \operatorname{IgM}$, and $\operatorname{IgA}$ (Conley et al. 1999). The clinical presentation of antibody deficiency is extremely heterogeneous, involving common clinical manifestations such as recurrent infections or comorbid autoimmune disease. Immunodeficiency and autoimmune diseases were previously considered to be completely opposite conditions, yet there is a high degree of overlap regarding genetic background. In fact, one study reported that approximately $26.2 \%$ of primary immunodeficiency patients have autoimmune symptoms (Fischer et al. 2017). In general, autoimmune manifestation in these patients predicts a poor prognosis, though the specific pathogenic mechanism is still unclear. In this study, we carried out whole-exome sequencing for a Chinese Han male patient with hypogammaglobulinemia and autoimmune manifestations to better understand the pathogenesis of antibody deficiency with concomitant autoimmune symptoms.

\section{Case Presentation}

The patient was a 29-year-old Chinese Han male who was admitted to the Department of Rheumatology and Immunology, West China Hospital in July 2018 with chief symptoms being repeated cough, fever, and chest tightness. At 2 years of age, the patient experienced sensorineural

Received March 19, 2021; revised and accepted July 26, 2021. Published online October 20, 2021; doi: 10.1620/tjem.255.143.

Correspondence: Yi Liu, Department of Rheumatology and Immunology, West China Hospital, Sichuan University, No. 37, Guoxue

Alley, Chengdu, Sichuan, 610041 China.

e-mail: yi2006liu@163.com

Tony N. Marion, Department of Rheumatology and Immunology, West China Hospital, Sichuan University, No. 37, Guoxue Alley, Chengdu, Sichuan 610041, China; Department of Microbiology, Immunology, and Biochemistry, University of Tennessee Health Science

Center, 910 Madison Ave, Memphis, TN 38163, USA.

e-mail: tmarion@uthsc.edu

(C)2021 Tohoku University Medical Press. This is an open-access article distributed under the terms of the Creative Commons Attribution-NonCommercial-NoDerivatives 4.0 International License (CC-BY-NC-ND 4.0). Anyone may download, reuse, copy, reprint, or distribute the article without modifications or adaptations for non-profit purposes if they cite the original authors and source properly.

https://creativecommons.org/licenses/by-nc-nd/4.0/ 
Table 1. Medical history.

\begin{aligned} & \hline Age \multicolumn{1}{c}{ Diseases } \\ & \hline 2 years Sensorineural hearing loss of unknown cause \\ & 21 years Recurrent facial acne \\ & 23 years Perianal abscess with anal fistula \\ & 26 years Pulmonary tuberculosis, erythema induratum \\ & 27 years Bronchiectasis with infection \\ & 29 years Pulmonary infection, immunosuppression \\ & 31 years Pulmonary tuberculosis, idiopathic thrombocytopenic purpura \\ & \hline\end{aligned}

Table 2. Immune profiles.

\begin{tabular}{|c|c|c|c|}
\hline & \multicolumn{3}{|c|}{ Age } \\
\hline & 29 years & 30 years & 31 years \\
\hline \multicolumn{4}{|l|}{ Immunoglobulins (reference range) } \\
\hline $\mathrm{IgG}, \mathrm{mg} / \mathrm{dL}(8,000-15,500)$ & 8,550 & 13,500 & 19,400 \\
\hline $\operatorname{IgA}, \mathrm{mg} / \mathrm{dL}(836-2,900)$ & 436 & 672 & 893 \\
\hline IgM, mg/dL (700-2,200) & 1,040 & 1,050 & 1,840 \\
\hline $\operatorname{IgE}, \mathrm{IU} / \mathrm{ml}(5-150)$ & ND & 11.30 & 7.96 \\
\hline \multicolumn{4}{|l|}{ Lymphocytes (reference range) } \\
\hline CD3+, cells/mL $(941-2,226)$ & 636 & 309 & 665 \\
\hline CD3+CD4+, cells/mL $(471-1,220)$ & 168 & 70 & 136 \\
\hline CD3+CD8+, cells/mL $(303-1,003)$ & 416 & 215 & 449 \\
\hline CD19+, cells/mL (175-332) & ND & 4 & 8 \\
\hline NK, \% $(9.26 \%-23.92 \%)$ & ND & 2.5 & ND \\
\hline \multicolumn{4}{|l|}{ Hemogram, $\times 10^{9}$ cells/L (reference range) } \\
\hline Monocytes (0.1-0.6) & 0.01 & 0 & 0.02 \\
\hline Neutrophils (1.8-6.3) & 7.41 & 3.62 & 2.85 \\
\hline Platelets (100-300) & 92 & 111 & 13 \\
\hline \multicolumn{4}{|l|}{ Vaccine titers } \\
\hline Tetanus toxoid, IU/ml (positive, $>0.1$ ) & Negative & ND & ND \\
\hline Tuberculosis antibody & Negative & ND & ND \\
\hline
\end{tabular}

ND, Not Done.

hearing loss of unknown cause; he developed recurrent pulmonary infections after the age of 20 (Table 1). Physical examination revealed thickened breath sound and moist rales in both lung bases. The bronchial symptoms were accompanied by redness and swelling of the skin of both lower limbs, with a flaky, red rash; some of the rash lesions had blistering. Hemogram analysis showed a white blood cell (WBC) count of $7.91 \times 10^{9} / \mathrm{L}$, with neutrophils accounting for $81.3 \%$. Immunological analysis detected a markedly decreased serum IgA level (436 mg/dL), though levels of IgG and IgM were normal (Table 2). Absolute T and $\mathrm{B}$ cell counts were significantly decreased (Table 2). Antinuclear antibody (titer 1:320) and anti-Ro/SSA $52 \mathrm{kDa}$ were positive. A chest CT scan revealed scattered bilateral infective lesions with bilateral pleural thickening, adhesion, and increased mediastinal small lymph nodes, as well as a small amount of pericardial effusion. Sputum culture was positive for Candida albicans. The patient's parents were in good health, with no history of any particular disease.

The patient was treated with antibiotics for bacterial and fungal infections, pulverization inhalation to dispel phlegm, and immunoglobulin $(400 \mathrm{mg} / \mathrm{kg})$ during hospitalization. The level of IgA was still low after intravenous immunoglobulin treatment, but $\mathrm{IgG}$ and $\operatorname{IgM}$ remained normal. Due to repeated infections and persistent low levels of IgA (448 mg/dL), hypogammaglobulinemia (IgA) was initially considered as the clinical possibility. The patient's condition subsequently improved, and he was discharged. After discharge, the patient was regularly followed up monthly in our outpatient clinic. In August 2018 and October 2019, he was hospitalized again for idiopathic thrombocytopenic purpura and pulmonary infection with Mycobacterium tuberculosis. After exclusion of myelodysplastic syndrome by bone marrow aspiration, the patient was discharged after anti-tuberculosis treatment and immunoenhancement treatment. The number of follow-up visits 
D07_XX1231_18C017643_GATA2-chr3-128200721_R603-E3_R.ab1 C $T$ T $T$ T A T G G C $\downarrow$

(A)

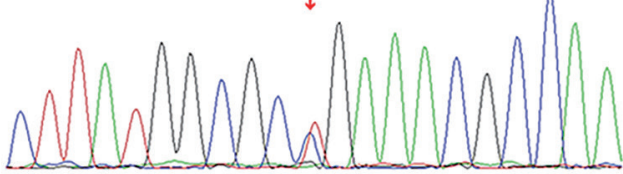

F07_XX1231_18C017644_GATA2-chr3-128200721_R603-E3_R.ab1

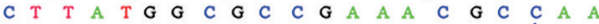

(B)

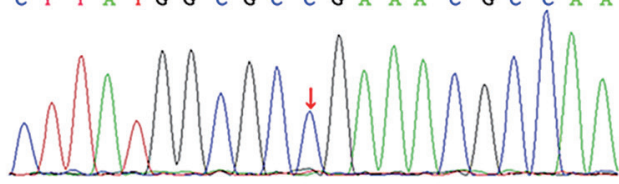

(C)

H07_XX1231_18C017645_GATA2-chr3-128200721_R603-E3_R.ab1

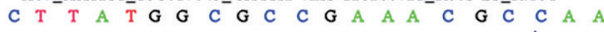

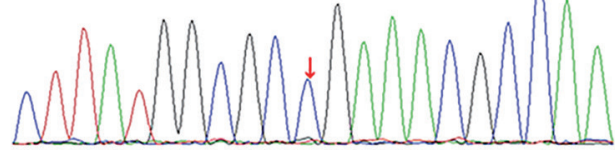

(D)

D06_XX1231_18C017643_STATSB-chr17-40359729_R501-A7_R_QX_D06.ab1

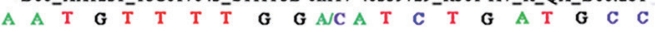

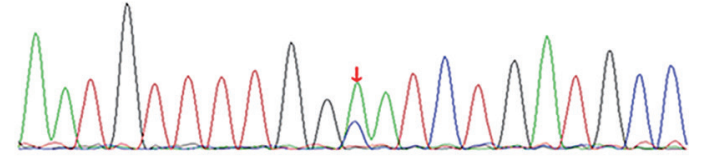

E06_XX1231_18C017644_STAT SB-chr17-40359729_R501-A7_R_QX_E06.ab1

(E)

$\begin{array}{llllllllllllllllllllll}A & A & T & G & T & T & T & T & G & G & A & A & T & C & T & G & A & T & G & C & C\end{array}$

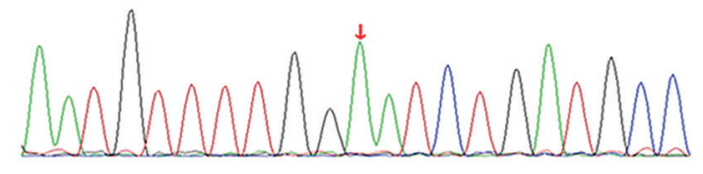

(F)
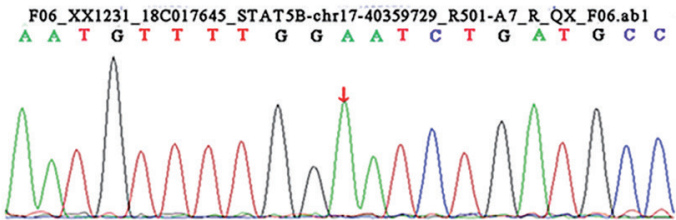

Fig. 1. Sanger sequencing validated the mutations at the two SNP sites.

(A-C) GATA2. (A) c. $1084 \mathrm{C}>\mathrm{T}$ nonsense mutation occurred at chr3-128200721 (GATA2) in the patient (heterozygous mutation) (indicated by an arrow). (B) Father of the patient. (C) mother of the patient.

(D-F) STAT5B. (D) c.1924A $>C$ missense mutation occurred at chr17-40359729 (STAT5B) in the patient (heterozygous mutation) (indicated by an arrow). (E) Father of the patient. (F) Mother of the patient.

was reduced during the novel coronavirus outbreak; his physical condition had recovered very well at the last follow-up visit to the Outpatient Department of our hospital in June 2020.

Whole-exome sequencing was performed for the patient and his parents, revealing two spontaneous heterozygous mutations in GATA2 (c.1084C $>$ T) and STAT5B (c.1924A $>$ C). The SNPs were validated as mutations by Sanger sequencing (Fig. 1).

The study protocol was approved by the Ethics
Committee of West China Hospital, Sichuan University. All participants provided their written informed consent for the study.

\section{Discussion}

Although the coexistence of immunodeficiency (a hypoimmune state) and autoimmunity (a hyperimmune state) seems paradoxical, it is common for them to coexist clinically. However, primary immunodeficiency with autoimmune symptoms tends to be more difficult to treat and has a worse prognosis. In the current report, the patient harbored the GATA2 mutation c. $1084 \mathrm{C}>\mathrm{T}$, which causes a cytosine to thymine change at nucleotide position 1,084, resulting in an amino acid change to a stop codon (p. R362X). Based on bioinformatic software SIFT, PolyPhen_2 and REVEL, this nonsense mutation has a predicted unknown effect on GATA2 protein function. Nevertheless, according to ACMG (Chinese Board of Genetic Counseling, http://acmg.cbgc.org.cn) guidelines, the mutation is pathogenic and reportedly related to myelodysplastic syndrome (Chen et al. 2019). The zinc finger transcription factor GATA2 affects cell fate in multiple tissue types by recognizing the common sequence of GATA in target gene promoters. In addition to its role in the development of hematopoietic stem cells, GATA2 has been shown to be an important regulator of the adult stem-cell pool. In addition, a lower dose of the GATA2 gene negatively affected proliferation and survival of primitive cells, though the differentiation or self-renewal ability of the remaining stem cells did not change (Rodrigues et al. 2005). Moreover, GATA2 mutations are associated with monocyte depletion, dendritic cell and B cell defects, myeloproliferation, immunodeficiency, and problems of the vascular and lymphatic systems (Hsu et al. 2011). Therefore, immunodeficiency caused by GATA2 dysfunction or insufficiency may result in infections, including mycobacterial, viral, and fungal infections. In one case report, a patient diagnosed with common variable immunodeficiency early due to hypogammaglobulinemia and defective antibody response was found to carry a heterozygous GATA2 mutation, with significantly decreased monocyte and lymphocyte counts during adolescence (Chou et al. 2014). Our case was mainly characterized by repeated infections, which is consistent with previous reports. In addition, Hoshino et al. (2019) found that GATA2 deficiency can lead to sensorineural hearing loss in mice, which may explain the hearing impairment in our patient.

In addition, we detected a missense mutation in one copy of STAT5B in the present patient, causing nucleotide 1,924 to change from adenine to cytosine, which results in amino acid 642 changing from asparagine to histidine (p.N642H). This STAT5B mutation (p.N642H) has been found in over 90 leukemia and lymphoma patients (Pham et al. 2018). According to ACMG guidelines, the mutation is preliminarily considered likely pathogenic. A previous study reported that patients with mutations in STAT5B often 
develop infections, autoimmune manifestations, eczemalike manifestations, and growth hormone insensitivity syndrome (Kofoed et al. 2003), but manifestations such as eczema and growth deficiency were not observed in our patient. Although study had proposed that STAT5B is related to development of primary immunodeficiency (Caldirola et al. 2018), we do not have enough evidence on the pathogenicity of STAT5B and more work is worth exploring in the future.

In conclusion, our study enriches our understanding of the pathogenic genes of antibody deficiency disorders, suggesting that coinheritance of mutations in immunoregulatory genes may affect the pathogenesis of primary immunodeficiency with autoimmunity. Further research with a large patient cohort is needed to clarify the interactive pathogenic roles of GATA2 and $S T A T 5 B$, especially when autoimmunity is concurrent.

\section{Acknowledgments}

This work was supported by the National Key Research and Development Program of China (grant number 2016YFC0906201) and the 1.3.5 Project for Disciplines of Excellence, West China Hospital, Sichuan University (grant number ZYGD18015). These sponsors had no role in the study design; collection, analysis and interpretation of data; writing of the report; or decision to submit the article for publication. We would like to thank the family of the patient for their kind cooperation and permission to publish this paper.

\section{Author Contributions}

Z.L. was responsible for the conception and design of the study, collection and interpretation of the clinical data, and for drafting of the manuscript. P.Q. and Y.Z. were responsible for suggestions and data acquisition and for revising the manuscript. Y.L. and T.M. participated in the clinical evaluation of the case and in critically revising the manuscript. All authors read and approved the final manuscript.

\section{Conflict of Interest}

The authors declare no conflict of interest.

\section{References}

Caldirola, M.S., Rodriguez Broggi, M.G., Gaillard, M.I.,
Bezrodnik, L. \& Zwirner, N.W. (2018) Primary immunodeficiencies unravel the role of IL-2/CD25/STAT5b in human natural killer cell maturation. Front. Immunol., 9, 1429.

Chen, L.L., He, X.L. \& Chen, K.K. (2019) Congenital deafmutism with pale complexion and anemia for 1 year in a school-aged girl. Zhongguo Dang Dai Er Ke Za Zhi, 21, 1105-1109.

Chou, J., Lutskiy, M., Tsitsikov, E., Notarangelo, L.D., Geha, R.S. \& Dioun, A. (2014) Presence of hypogammaglobulinemia and abnormal antibody responses in GATA2 deficiency. $J$. Allergy Clin. Immunol., 134, 223-226.

Conley, M.E., Notarangelo, L.D. \& Etzioni, A. (1999) Diagnostic criteria for primary immunodeficiencies. Representing PAGID (Pan-American Group for Immunodeficiency) and ESID (European Society for Immunodeficiencies). Clin. Immunol., 93, 190-197.

Fischer, A., Provot, J., Jais, J.P., Alcais, A. \& Mahlaoui, N.; members of the CEREDIH French PID study group (2017) Autoimmune and inflammatory manifestations occur frequently in patients with primary immunodeficiencies. $J$. Allergy Clin. Immunol., 140, 1388-1393 e1388.

Hoshino, T., Terunuma, T., Takai, J., Uemura, S., Nakamura, Y., Hamada, M., Takahashi, S., Yamamoto, M., Engel, J.D. \& Moriguchi, T. (2019) Spiral ganglion cell degenerationinduced deafness as a consequence of reduced GATA factor activity. Genes. Cells, 24, 534-545.

Hsu, A.P., Sampaio, E.P., Khan, J., Calvo, K.R., Lemieux, J.E., Patel, S.Y., Frucht, D.M., Vinh, D.C., Auth, R.D., Freeman, A.F., Olivier, K.N., Uzel, G., Zerbe, C.S., Spalding, C., Pittaluga, S., et al. (2011) Mutations in GATA2 are associated with the autosomal dominant and sporadic monocytopenia and mycobacterial infection (MonoMAC) syndrome. Blood, 118, 2653-2655.

Kofoed, E.M., Hwa, V., Little, B., Woods, K.A., Buckway, C.K., Tsubaki, J., Pratt, K.L., Bezrodnik, L., Jasper, H., Tepper, A., Heinrich, J.J. \& Rosenfeld, R.G. (2003) Growth hormone insensitivity associated with a STAT5b mutation. N. Engl. J. Med., 349, 1139-1147.

Notarangelo, L., Casanova, J.L., Fischer, A., Puck, J., Rosen, F., Seger, R. \& Geha, R.; International Union of Immunological Societies Primary Immunodeficiency diseases classification committee (2004) Primary immunodeficiency diseases: an update. J. Allergy Clin. Immunol., 114, 677-687.

Pham, H.T.T., Maurer, B., Prchal-Murphy, M., Grausenburger, R., Grundschober, E., Javaheri, T., Nivarthi, H., Boersma, A., Kolbe, T., Elabd, M., Halbritter, F., Pencik, J., Kazemi, Z., Grebien, F., Hengstschläger, M., et al. (2018) STAT5BN642H is a driver mutation for T cell neoplasia. J. Clin. Invest., 128, 387-401.

Rodrigues, N.P., Janzen, V., Forkert, R., Dombkowski, D.M., Boyd, A.S., Orkin, S.H., Enver, T., Vyas, P. \& Scadden, D.T. (2005) Haploinsufficiency of GATA-2 perturbs adult hematopoietic stem-cell homeostasis. Blood, 106, 477-484. 